\section{OPEN ACCESS}

Edited by:

Fenglin Liu,

Fudan University, China

Reviewed by:

Zhengrong Li,

First Affiliated Hospital of Nanchang

University, China

Jun Li,

St. Jude Children's Research Hospital,

United States

*Correspondence:

Seong-Ho Kong

seongho.kong@snu.ac.kr

Chunchao Zhu

zcczmy@hotmail.com

${ }^{\dagger}$ These authors have contributed equally to this work

Specialty section:

This article was submitted to Gastrointestinal Cancers,

a section of the journal

Frontiers in Oncology

Received: 24 August 2020 Accepted: 06 November 2020

Published: 08 January 2021

Citation:

Zhang Y, Yu F, Ni B, Li Q, Bae S-W, Choi J-H, Yang $H-K$, Kong S-H and Zhu C (2021) The RNA-Binding

Protein DDX18 Promotes Gastric Cancer by Affecting the Maturation of MicroRNA-21.

Front. Oncol. 10:598238. doi: 10.3389/fonc.2020.598238

\title{
The RNA-Binding Protein DDX18 Promotes Gastric Cancer by Affecting the Maturation of MicroRNA-21
}

\author{
Yeqian Zhang ${ }^{1,2 \dagger}$, Fengrong $\mathrm{Yu}^{1 \dagger}$, Bo $\mathrm{Ni}^{1,2 \dagger}$, Qing $\mathrm{Li}^{2}$, Seong-Woo Bae ${ }^{3}$, Jong-Ho Choi ${ }^{3}$, \\ Han-Kwang Yang ${ }^{3,4}$, Seong-Ho Kong ${ }^{3,4^{*}}$ and Chunchao Zhu ${ }^{1 *}$ \\ ${ }_{1}^{1}$ Department of Gastrointestinal Surgery, Renji Hospital, School of Medicine, Shanghai Jiao Tong University, Shanghai, \\ China, 2 State Key Laboratory of Oncogenes and Related Genes, Shanghai Cancer Institute, Renji Hospital, School of \\ Medicine, Shanghai Jiao Tong University, Shanghai, China, ${ }^{3}$ Department of Surgery, Seoul National University Hospital, \\ Seoul, South Korea, ${ }^{4}$ CancerResearch Institute, Seoul National University, Seoul, South Korea
}

Objectives: The noncoding RNAs (ncRNAs) play important roles in gastric cancer. Most studies have focused on the functions and influence of ncRNAs, but seldom on their maturation. DEAD box genes are a family of RNA-binding proteins that may influence the development of ncRNAs, which attracted our attention. By combining a small sample for high-throughput gene microarray screening with large samples of The Cancer Genome Atlas (TCGA) data and our cohort, we aimed to find some gastric cancer-related genes. We evaluated the clinical significance and prognostic value of candidate gene DDX18, which is overexpressed in gastric cancer tissues. To provide a theoretical basis for the development of new therapeutic targets for the treatment of gastric cancer, we investigated its effect on the malignant biological behavior of gastric cancer in vitro and in vivo, and also discuss its mechanism of action.

Methods: (i) The differential profiling of mRNA expression in five pairs of gastric cancer and adjacent normal tissues was studied by Arraystar Human mRNA Microarray. By combining this with TCGA data and our cohort, we finally filtered out DDX18, which was upregulated in gastric cancer tissues, for further investigation. (ii) The protein expression of DDX18 was detected by immunohistochemistry staining. Then the relationship between the DDX18 expression level and the clinicopathological data and prognosis was analyzed. (iii) A CCK-8 assay and colony formation assay were used to evaluate the effect of DDX18 on cell growth and proliferation in vitro. A transwell assay was also performed to examine the migration and invasion of gastric cancer cells. Cell apoptosis was analyzed by using a fluorescein isothiocyanate-annexin V/propidium iodide double-staining assay. To identify the role of DDX18 in the tumorigenic ability of gastric cancer cells in vivo, we also established a subcutaneous gastric cancer xenograft model. Coimmunoprecipitation, small RNAseq, and western blotting were performed to explore the mechanism of action of DDX18 in gastric cancer. A patient-derived xenograft (PDX) model was used to confirm the effect of DDX18 in gastric cancer tissues. 
Result: (i) DDX18 was upregulated in gastric cancer tumor tissues from a TCGA database and our cohort. The expression of DDX18 was also closely related to tumor volume, Borrmann classification, degree of tumor differentiation, cancer embolus, lymph node metastasis, and TNM stage. (ii) DDX18 could promote cell proliferation, migration, and invasion and inhibit cell apoptosis in vivo and in vitro. (iii) DDX18 could promote the maturation of microRNA-21 through direct interaction with Drosha, decreasing PTEN, which could upregulate the AKT signaling pathway. (iv) The PDX model showed that DDX18 could promote the proliferation of gastric cancer tissues by means of the PTENAKT signaling pathway.

Conclusions: (i) DDX18 can be treated as a molecular marker to assess the prognosis of patients with gastric cancer. (ii) DDX18 could be a potential therapeutic target in gastric cancer.

Keywords: gastric cacner, ncRNA (noncoding RNA), DEAD box family, PTEN (phosphatase and tensin homolog deleted on chromosome 10), Drosha-independent miRNA

\section{INTRODUCTION}

As the third most deadly cancer worldwide, gastric cancer is a serious threat to human health (1). While early gastric cancer has a good cure rate, advanced or late gastric cancer often has a poor prognosis. Therefore, it is very important to find specific biomarkers to evaluate and predict the occurrence and metastasis of gastric cancer and assess treatments to improve the diagnosis and treatment of this disease.

An increasing number of studies have shown that noncoding RNAs (ncRNAs) such as microRNAs and long noncoding RNAs (lncRNAs) play an important role in the occurrence and development of gastric cancer. However, these studies have mainly focused on the regulation and influence of microRNAs and $\operatorname{lncRNAs}$ on downstream genes. How ncRNAs are regulated is relatively unknown.

The DDX (DEAD-box gene) family is a family of RNA helicases that was first proposed by Patrick Linder in 1989 (2, 3). The DEAD box has nine conserved regions (Q, I, Ia, Ib, II-VI) and region II, which includes D (Asp), E (Glu), A (Ala), and D, which has been recognized as a characteristic sequence. These proteins are obtained by hydrolysis of ATP and then release RNAs to regulate cellular processes such as translation initiation, nuclear and mitochondrial splicing, and ribosome and splice packaging. Previous studies have suggested that the DDX family members are expressed abnormally in tumor tissues, which is related to tumor occurrence, development, metastasis, and invasion (4-6).

The expression levels of mature microRNAs are mainly determined by a critical rate-limiting step: the processing of premature microRNAs (pri-microRNAs and pre-microRNAs) (2). The Drosha microprocessor, whose core components are Drosha and DGCR8, functions first in microRNA maturation (7). Nevertheless, neither Drosha nor DGCR8 can recognize the indicated pri-microRNAs with any specificity (8). Regulatory components are needed in the microprocessor to offer specificity for recruiting and processing pri-microRNAs, accounting for the finding that the same primary transcript could generate diverse expression levels of mature microRNA.

In this study, we first used gene chip technology to explore the differentially expressed genes in gastric cancer. Further analysis of the clinical and pathological data identified the high expression of DDX18 in gastric cancer tissues. We performed cell function experiments to study the role of DDX18 in gastric cancer. We also explored its molecular mechanism by means of molecular cytology technology and animal experiments to elucidate DDX18 and to determine its role in the occurrence and development of gastric cancer.

\section{MATERIALS AND METHODS}

\section{Patients and Tissue Samples}

Thirty-seven pairs of fresh gastric cancer and control normal gastric tissue specimens were obtained during surgery carried out on 37 patients from January 2013 to August 2014. All 37 patients underwent resection of primary gastric cancer at Renji Hospital, School of Medicine, Shanghai Jiao Tong University, Shanghai, China. Resected cancer tissues and paired noncancerous tissues were immediately cut and frozen in liquid nitrogen, and kept at $80^{\circ} \mathrm{C}$ until RNA and DNA extraction for quantitative real-time polymerase chain reaction (PCR).

For the assessment of immunoreactivity and the prognostic value of DDX18 in gastric cancers, the inclusion criteria for patients with gastric cancer were as follows: (i) a distinct pathological diagnosis of gastric adenocarcinoma; (ii) no radiotherapy, chemotherapy, or other anticancer therapies prior to surgery; (iii) primary tumor resection, including radical gastrectomy and palliative gastrectomy; and (iv) availability of complete clinicopathological and follow-up data.

A total of 585 paraffin-embedded tissue samples that met the above criteria were collected from patients with gastric cancer at the Department of Gastrointestinal Surgery, Renji Hospital, from January 2006 to December 2011 for tissue microarray 
construction and immunohistochemistry (IHC) staining. The overall survival (OS) is calculated from the date of tumor resection until death.

\section{Cell Lines and Cell Cultures}

Cells from the human gastric cancer cell lines SGC-7901, NCIN87, HGC27, MGC-803, BGC-823, and AGS were purchased from the Institute of Biochemistry and Cell Biology, Chinese Academy of Sciences, Shanghai, China. All the cells were cultured in Roswell Park Memorial Institute (RPMI) 1640 medium (Invitrogen, Carlsbad, CA, USA). The medium contained $10 \%$ fetal bovine serum and $1 \%$ penicillin/ streptomycin. All cells were incubated in a cell incubator under $5 \% \mathrm{CO}_{2}$ at $37^{\circ} \mathrm{C}$.

\section{Lentivirus Transfection}

Genomeditech (Shanghai, China) assisted in the design and production of DDX18 short hairpin RNA (shRNA).

Six-well plates were prepared and inoculated with the appropriate gastric cancer cells, with the adherent cells occupying about $50 \%$ of the total area of the plates. The appropriate amount of lentivirus was added to each well according to the multiplicity of infection value of gastric cancer cells. The cells were screened with antibiotics recommended by Genomeditech, and the transfection efficiency was measured by fluorescence quantitative PCR or western blot. The target sequences of DDX18 were: sh-1, sense: 5'-3', GCAGCGGAACCTAAAGTTT; sh-2, sense: 5'-3', GCA TACCTATGGCTTGATA; sh-control, sense: 5'-3', TT CTCCGAACGTGTCACGT.

\section{RNA Extraction and Quantitative Real- Time PCR}

Total RNA was extracted with Trizol and reverse-transcribed into complementary DNA (cDNA) by PrimeScript ${ }^{\mathrm{TM}}$ (Takara Biomedical Technology, Beijing, China). Using glyceraldehyde 3phosphate dehydrogenase (GAPDH) as the internal reference, realtime PCR analysis was conducted using an Applied Biosystems 7500 Real-Time PCR System (biological system), and the relative expression level of the target genes was calculated by the $2^{-\Delta \Delta \mathrm{Ct}}$ method. The primer sequences were: DDX18: $5^{\prime}-3^{\prime}, \mathrm{F}-$ ATGTCACACCTGCCGATGAAA, R-CCCTGAAAC TTTAGGTTCCGC; GAPDH: 5'-3', F-GGAGCGAGAT CCCTCCAAAAT, R-GGCTGTTGTCATACTTCTCATGG.

\section{Western Blotting}

Gastric cancer cells were lysed by radioimmunoprecipitation assay (RIPA) buffer (Beyotime, Beijing, China) and protease inhibitors (Roche, CA, USA). Then, $10 \%$ sodium dodecyl sulfate glue was used for electrophoresis and electrorotation to transfer the protein to the nitrocellulose (NC) membrane. The NC membrane was blocked for 1 hour at room temperature in Tris-buffered saline containing 5\% skimmed milk. After incubation with primary and secondary antibodies, electrochemiluminescence was used to obtain bands (antibodies: DDX18 Abcam ab70527; P-AKT cell signaling SER473; AKT 10174-2-AP; PTEN Abcam ab32199).

\section{Coimmunoprecipitation}

We linked three labels (FLAG-DDX18, HA-Drosha, HisDGCR8) to the indicated proteins. The prepared cells transfected with FLAG-DDX18, HA-Drosha, and His-DGCR8 plasmids were collected for nuclear protein extraction followed by coimmunoprecipitation. A nuclear protein extraction kit (P0027, Beyotime) was used according to the manufacturer's protocol. In brief, alternate vortexing and centrifugation combined with the extraction kit were used to separate the total proteins into nuclear and cytoplasmic proteins. At the same time as the cell nuclear proteins were prepared, protein A/G magnetic beads (B23201; Bimake, Shanghai, China) were preincubated on a spinning wheel at $4^{\circ} \mathrm{C}$ for 30-60 minutes and washed three times with PBS. The antibody complex was then suspended in the nuclear protein solution. After the protein solution was fully combined with the magnetic bead-antibody complex, the extraction buffer was washed three times. Magnetic separation was performed by heating. The immunoprecipitate was collected and western blotting was performed.

\section{Cell Proliferation}

The proliferation capacity of gastric cancer cells was determined by cell count kit 8 (CCK-8; Beyotime, China). After transfection with DDX18 shRNA, gastric cancer cells were inoculated in 96well plates, with about 2000 cells per well. Under dark conditions, each chamber was incubated for 1 hour with $10 \mu \mathrm{l}$ CCK- 8 reagent and the optical density was measured at a wavelength of $450 \mathrm{~nm}$ with a SpectraMax Plus 384 (Molecular Devices). Six-well plates were used to lay about 500 gastric cancer cells in each well, and then the cells were allowed to grow for about 2 weeks. After washing twice with phosphate-buffered saline (PBS), cells were fixed in 4\% paraformaldehyde for 15 minutes, and $0.5 \%$ crystal violet staining was performed for a further 15 minutes. The number of gastric cancer cell clones in different groups was calculated.

\section{Detection of Apoptosis}

For the cell apoptosis assay, $20 \times 10^{5}$ cells per well were cultured under serum deprivation in six-well plates. Adherent cells were detached with $0.25 \%$ trypsin without ethylenediaminetetraacetic acid in $1 \times$ PBS. Cells were harvested in complete RPMI 1640 and centrifuged at 1000 r.p.m. for 5 minutes. Each batch of cells was washed with $1 \times \mathrm{PBS}$ and stained with $50 \mathrm{mg} / \mathrm{ml}$ propidium iodide (PI) and annexin V-fluorescein isothiocyanate according to the manufacturer's instructions. The percentage of annexin $\mathrm{V}(+)$ and PI (-) cells was analyzed by flow cytometry.

\section{Transwell Assay}

A transwell chamber was prepared with $600 \mu \mathrm{l}$ serum-free RPMI 1640 medium added to each well, followed by $5 \times 10^{5}$ cells. The culture medium containing $20 \%$ serum was used in the lower layer. After 24 hours, the chamber was washed twice with PBS, $4 \%$ paraformaldehyde was used to fix the cells for 15 minutes, crystal violet was used for staining for 15 minutes, and the number of cells passing through the chamber was counted under a microscope. 


\section{Luciferase Reporter Gene}

The DDX18 gene, PTEN gene 3'-untranslated region (UTR) target clone (CmiT010099-MT05), and negative control clone (CmiT000001-MT05) were purchased from GengCopia Company, USA. For the secretory double luciferase reporter gene analysis, 293T cells were inoculated in six-well plates and cotransfected when the cell confluence reached 50\%. The culture medium was changed to whole medium 24 hours after transfection and incubation continued. The supernatant of the cell culture solution was carefully collected 48 hours after transfection and packed into two tubes, each containing $200 \mu \mathrm{L}$, which was used for the detection of Gaussia luciferase (GLuc) and secretory alkaline phosphatase (SeAP), respectively. The supernatant used to detect SeAP was placed in a water bath at $65^{\circ} \mathrm{C}$ for 15 minutes; the working solution of $1 \times$ glucose was prepared and incubated in the dark at room temperature for 25 minutes; the working solution of $1 \times$ SeAP was prepared and incubated in the dark at room temperature for 10 minutes; $10 \mu \mathrm{l}$ of supernatant and $100 \mu \mathrm{l}$ of the corresponding working solution were added to each well of a 96-well plate, and multiples of three were used in each group. After incubation at room temperature for 1-10 minutes, the fluorescence was determined using a microplate reader. For data processing, with the SeAP fluorescence reading as the internal reference, the ratio of the GLuc fluorescence reading to the SeAP fluorescence reading was compared among the groups, and the mean values of three reading plates and three complex holes were taken.

\section{Immunohistochemistry and Staining Evaluation}

Paraffin sections were dewaxed with xylene, rehydrated by fractional ethanol, and antigens were extracted. The sections were blocked with $10 \%$ bovine serum albumin (BSA), incubated with primary antibody for 1 hour, and then incubated with secondary antibody for 30 minutes at room temperature. DAPI (4',6-diamino2-phenylindole hydrochloride; AppliChem, A4099) was used to stain the nucleus. An automatic fluorescence microscope (Nikon) was used for image observation and analysis. The tissue sections were assessed and graded by two independent investigators who were unaware of the clinicopathological factors. The staining intensity was 0 (negative), 1 (weak), 2 (medium), and 3 (strong).The degree of staining was stratified as $0(0 \%), 1$ (1$25 \%), 2(26-50 \%), 3(51-75 \%)$, and 4 (76-100\%), defined as the percentage of positive staining area in the total tumor invasion area. The final score of DDX18 expression was $0-7$. The samples were divided into two groups: low DDX18 expression ( $0-3$ points; IHC0 ) and high DDX18 expression (4-7 points; IHC-1,2).

\section{Cellular Immunofluorescence}

After immersion in 100\% alcohol, alcohol lamp burning, and ultraviolet irradiation, a glass slide was placed into a six-well plate, and gastric cancer cells were spread on the glass slide. The cells were allowed to grow to $30-50 \%$ of the area of the glass slide on the second day. Cells were fixed with $4 \%$ paraformaldehyde for 15 minutes and treated with $0.5 \%$ Triton X-100 for 1 minute. Nonspecific binding sites were blocked by $1 \%$ BSA. The primary antibody was incubated for 1 hour at room temperature and the secondary antibody for 30 minutes, and then treated with DAPI for 30 minutes for nuclear staining. Observation and analysis were conducted by automatic fluorescence microscope (Nikon).

\section{Animal Models}

To construct the subcutaneous tumor model of nude mice, $5 \times 10^{6}$ gastric cancer cells were injected into the left axilla of each nude mouse. After 4 weeks, the nude mice were killed, the subcutaneous tumor was removed, and its weight and volume were measured; $4 \%$ paraformaldehyde was used to store the tumor. All animal experiments were approved by the Ethics Committee of Renji Hospital.

\section{Statistical Analysis}

SPSS 22.0 (SPSS Inc., Chicago, IL, USA) was used to analyze and calculate all the data, and the value was the mean \pm SD. Student's $t$-test and the chi-squared test were used in the study. The Cox proportional hazard model was used for univariate and multivariate analysis to understand the factors affecting survival. $P<0.05$ was considered statistically significant.

\section{RESULTS}

\section{DDX18 Is Outstanding in Gastric Cancer Gene Chip Analysis}

The mRNA expression profile of the known protein in five cases of gastric cancer and adjacent tissues was analyzed by an Arraystar Human mRNA Microarray v2.0 gene chip. The GAPDH gene was used as the internal control, and the fold difference between the different groups of $>2$ or $<-2$ with $P<0.05$ was used for standard screening. In all the genes upregulated, five genes with the highest fold change were obtained, which were TWIST2, MET, HOXA13, DDX18, and PRND (Figure 1A).

\section{Differential Expression of DDX18 in gastric cancer tissues}

First, we assessed five pairs of gastric cancer tissues and matched paracancerous tissues by using gastric cancer gene chip technology. We used a fold change $>2$ and $P<0.05$ as the inclusion criteria, and DDX18 attracted our attention among the top differentially expressed genes (Figure 1A). In other words, the DDX family of genes caught our attention. Combining this with The Cancer Genome Atlas (TCGA) database, we focused on DDX18 as our target of interest (Figure 1B). Next, we examined DDX18 in 22 pairs of fresh gastric cancer tissues from a large sample set by quantitative PCR (Figure 2B) and western blot analyses (FigureS 2C, D), which confirmed that DDX18 was specifically highly expressed in gastric cancer tissues $(P<0.05)$. Similar results were verified in the TCGA database (Figure 2A).

Next, we detected the expression of DDX18 in 585 cases of gastric cancer by IHC. IHC-0 indicated negative DDX18 expression, and IHC-1 and IHC-2 indicated positive DDX18 expression, according to immunohistochemical grade (Figure 2E). Subsequently, we analyzed the relationship between the 

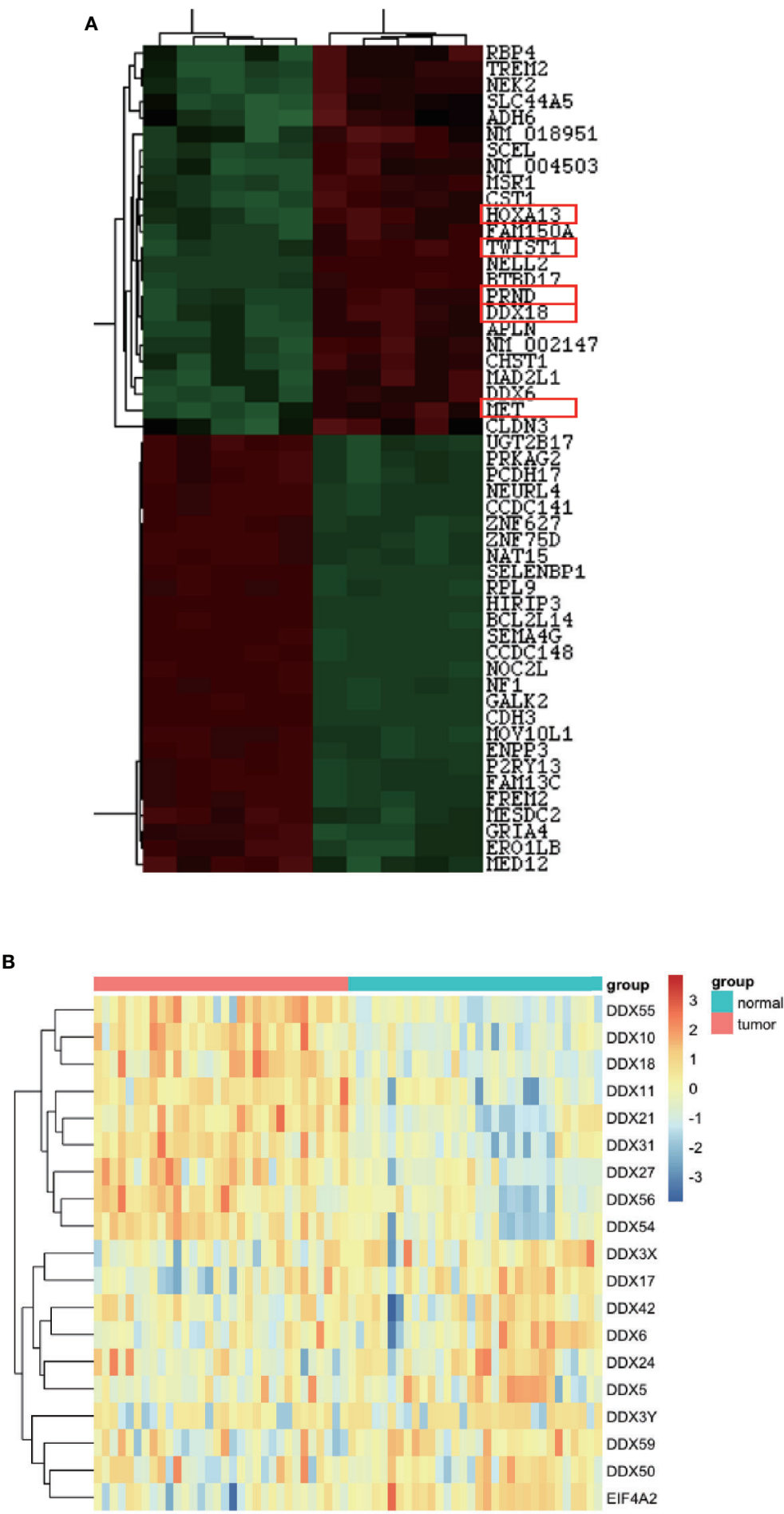

FIGURE 1 | DDX18 is highly expressed in gastric cancer. (A) DDX18 expression on a gene chip. (B) DDX gene family expressions in The Cancer Genome Atlas (TCGA) database.

DDX18 protein level and the clinicopathological features of gastric cancer in 585 cases of gastric cancer. The results of univariate analysis showed that positive DDX18 protein expression was present in $65.0 \%(380 / 585)$ of gastric cancer cases. The expression level of DDX18 was closely related to tumor location, tumor size, Borrmann classification, differentiation, intravascular tumor thrombus formation, nerve invasion, depth of invasion, lymph node metastasis, and TNM staging but not to age or gender. 
A

DDX18 expression from TCGA

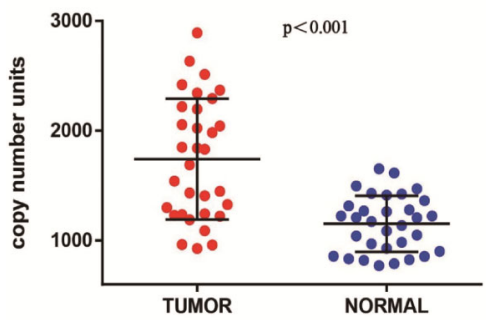

C
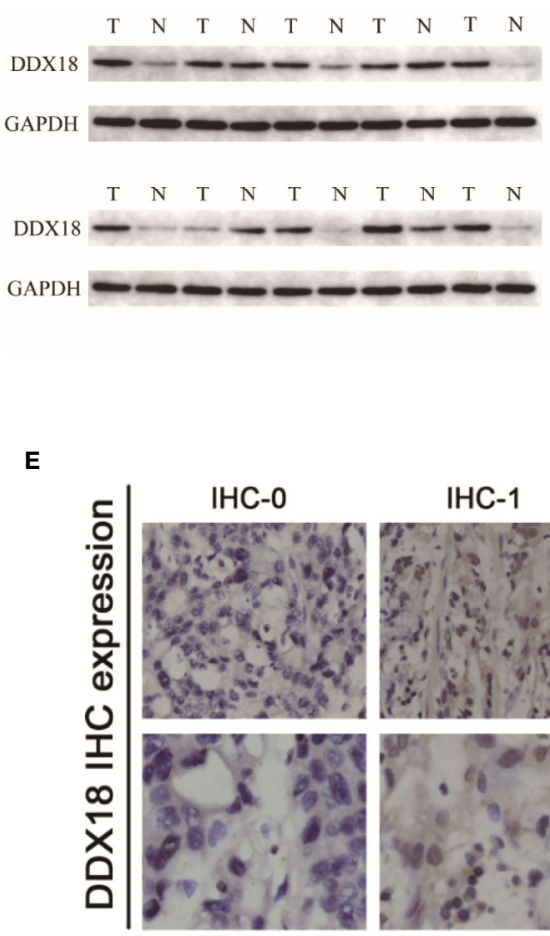

DDX18 mRNA expression

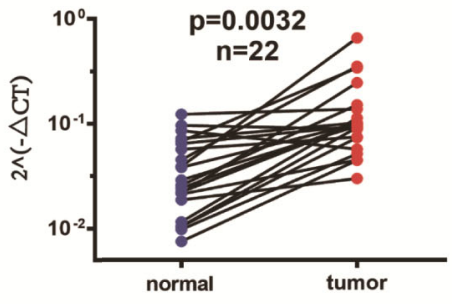

D

DDX18 expression of paired tissues

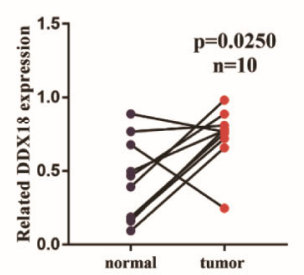

IHC-2
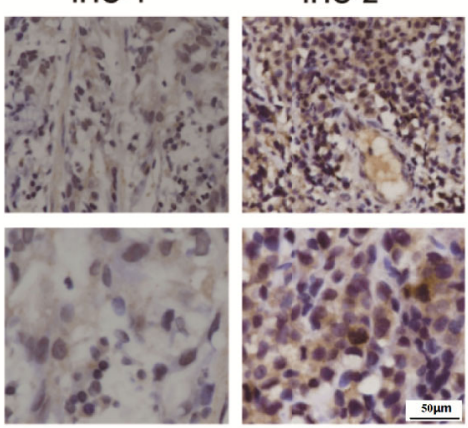

FIGURE 2 | DDX18 expressions in gastric cancer tissues. (A) DDX18 expressions from The Cancer Genome Atlas (TCGA). (B) DDX18 mRNA expression in 22 paired gastric cancer tissues. (C, D) DDX18 protein expressions in 10 paired gastric cancer tissues. (E) DDX18 expression by immunohistochemistry.

All patients were divided into the DDX18 high-expression group (a total of 380 patients with IHC-1 and IHC-2) and the DDX18 low-expression group (a total of 205 patients with IHC0 ), which were divided by DDX18 immunohistochemistry. The OS rates of the patients in the low-expression group were $90.6 \%$, $76.7 \%$, and $67.8 \%$. The OS rates of the patients with high expression were $88.9 \%, 63.1 \%$, and $54.7 \%$. The difference between the two groups in the first stage of gastric cancer was not significant, but in stage II and stage III gastric cancer significant differences were observed (Figures 3A-D).

The results showed that the degree of tumor differentiation, lesion site, Borrmann classification, tumor size, intravascular thrombosis, nerve invasion, depth of invasion ( $\mathrm{T}$ stage), lymph node metastasis (N stage), and DDX18 expression level had significant effects on the OS rate of 585 patients with gastric cancer, and the difference was significant $(P<0.05)$ (Table 1). The depth of invasion ( $\mathrm{T}$ stage), lymph node metastasis $(\mathrm{N}$ stage), and DDX18 expression were independent prognostic risk factors (Tables 2 and 3 ).

\section{Impact of DDX18 on Gastric Cancer Cell Lines}

We detected the expression of DDX18 in gastric cancer cell lines (Figure 4A). Then we designed two target sequences of shDDX18, and a mixture of two shRNAs was used in the following experiments. The proliferative activity of the AGS- 

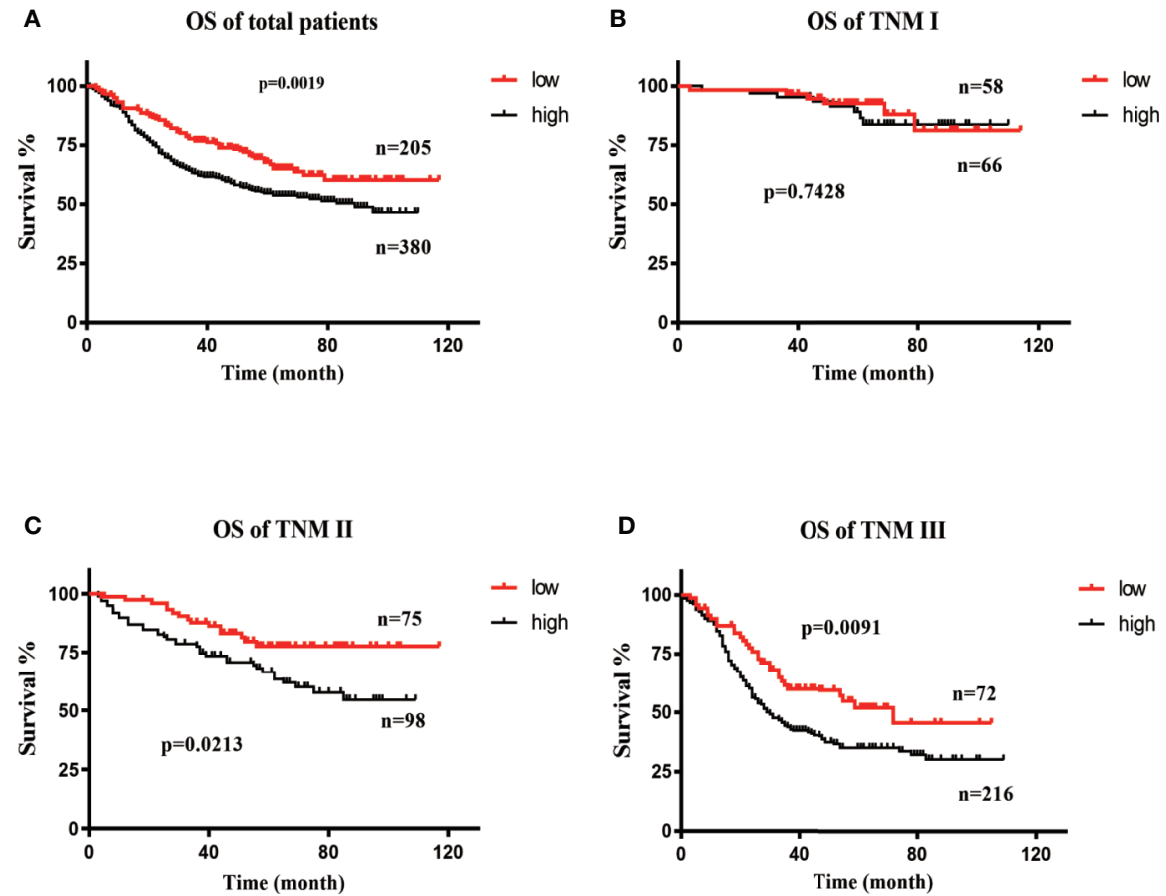

FIGURE 3 | Survival curve of DDX18. (A) Overall survival (OS) curve of DDX18. (B) Survival curve of patients with TNM stage (I) (C) Survival curve of patients with TNM stage II. (D) Survival curve of patients with TNM stage III.

sh-DDX18 cells and the control cells was compared by the CCK8 method. The $\mathrm{OD}_{450}$ values of each group were detected for five consecutive days. After 5 days, DDX18 knockdown significantly decreased the proliferation rate of tumor cells by $63.0 \%$ $(P<0.001)$ (Figure 4B). Subsequently, we compared the proliferative activity of the SGC-7901-OE cells and the control cells and found that exogenous DDX18 could significantly promote the growth of the SGC-7901 cells. The difference was significant $(P<0.001)$ (Figure 4C).

We found that the interference group's ability to form clones $(82.3 \pm 5.31)$ was significantly lower than that of the control group $(289.4 \pm 11.2)(P<0.01)$ (Figure $4 D)$. The results demonstrated that DDX18 has a significant effect on the proliferation of individual adherent cells.

The transwell results showed that the number of penetrating cells in the control group was $83.6 \pm 12.5$ cells/high-power field (HPF), which was significantly higher than that of the interference group $(26.6 \pm 4.3$ cells/HPF). The migration of the AGS cells was significantly weaker than that of the control group $(P<0.01)$ after stable knockdown of DDX18 gene expression (Figure 4E). Similar results were found in the BGC-823 cell line, which was another gastric cell line with high DDX18 expression (Figure S1).

In the cell apoptosis experiment, we used serum-free culture to induce apoptosis to study the effects of DDX18 on the absence of a nutrient supply. This enabled us to study the antiapoptotic ability.

The results showed that the average apoptotic rate of the interference group was $27.58 \pm 3.63 \%$ compared with
$7.03 \pm 1.44 \%$ for the control group. The proportion of apoptotic cells increased significantly $(P<0.01)$, suggesting that interference with the DDX18 gene in the AGS cells significantly reduced the antiapoptotic ability (Figure 4F). Similar results showed that the average apoptosis rate of the overexpression group was $16.09 \pm 2.24 \%$ after 48 hours of starvation, which was significantly lower than that of the control group $(28.64 \pm 4.33 \%, P<0.01)$, suggesting that the antiapoptotic ability of the SGC-7901 cells was significantly enhanced after overexpression of the DDX18 gene (Figure 4G).

\section{Interaction of DDX18 and Drosha}

In a previous study, we found that DDX18 is highly expressed in gastric cancer. Cell and animal experiments have confirmed that DDX18 promotes the proliferation of gastric cancer cells. However, the mechanism that DDX18 exerted in this process remains unclear: DDX families belong to a family of RNA helicases with conserved DEAD domains, which have been found to play a role in various tumors. In addition to the original functions of DDX18 and the DDX family, we speculated that DDX18 might be related to the formation and maturation of RNAs. Therefore, we hypothesized that DDX18 might be related to the maturation of microRNAs. Next, we constructed a DDX18-KD cell model and detected the expression of microRNAs after DDX18 knockdown by small RNA-seq. We found that the expression of microRNA-21 in the DDX18-KD cell lines decreased significantly (Figure 5C). Combined with the detection of 22 clinical samples, the results showed that the expression of microRNA-21 was positively correlated with 
TABLE 1 | Association of DDX18 expression with clinicopathological characteristics in 585 patients with gastric cancer.

\begin{tabular}{|c|c|c|c|c|c|c|c|}
\hline \multirow[t]{2}{*}{ Clinicopathological characteristic } & \multirow[t]{2}{*}{ No. of cases } & \multicolumn{4}{|c|}{ DDX18 expression } & \multirow[t]{2}{*}{$\chi^{2}$} & \multirow[t]{2}{*}{$P$-value } \\
\hline & & \multicolumn{2}{|c|}{$\begin{array}{c}\text { Negative } \\
n=205(35.0 \%)\end{array}$} & \multicolumn{2}{|c|}{$\begin{array}{c}\text { Positive } \\
n=380(65.0 \%)\end{array}$} & & \\
\hline \multicolumn{8}{|l|}{ Gender } \\
\hline Male & 400 & 139 & $34.8 \%$ & 261 & $65.3 \%$ & 0.048 & 0.827 \\
\hline Female & 185 & 66 & $35.7 \%$ & 119 & $64.3 \%$ & & \\
\hline \multicolumn{8}{|l|}{ Age (years) } \\
\hline$\leq 60$ & 273 & 94 & $34.4 \%$ & 179 & $65.6 \%$ & 0.084 & 0.772 \\
\hline$>60$ & 312 & 111 & $35.6 \%$ & 201 & $64.4 \%$ & & \\
\hline \multicolumn{8}{|l|}{ Tumor location } \\
\hline Upper & 76 & 22 & $28.9 \%$ & 54 & $71.1 \%$ & 967.9 & $<0.001^{\star \star *}$ \\
\hline Middle & 115 & 36 & $31.3 \%$ & 79 & $68.7 \%$ & & \\
\hline Lower & 309 & 116 & $37.5 \%$ & 193 & $62.5 \%$ & & \\
\hline Whole & 80 & 29 & $36.3 \%$ & 51 & $63.8 \%$ & & \\
\hline Residual & 5 & 2 & $40.0 \%$ & 3 & $60.0 \%$ & & \\
\hline \multicolumn{8}{|l|}{ Tumor size (cm) } \\
\hline$<5$ & 307 & 124 & $40.4 \%$ & 183 & $59.6 \%$ & 8.118 & $0.004^{\star \star}$ \\
\hline$\geq 5$ & 278 & 81 & $29.1 \%$ & 197 & $70.9 \%$ & & \\
\hline \multicolumn{8}{|l|}{ Borrmann $^{a}$} \\
\hline । & 33 & 13 & $39.4 \%$ & 20 & $60.6 \%$ & 509.1 & $<0.001^{\star \star \star}$ \\
\hline$\|$ & 76 & 29 & $38.2 \%$ & 47 & $61.8 \%$ & & \\
\hline III & 333 & 111 & $33.0 \%$ & 223 & $67.0 \%$ & & \\
\hline IV & 66 & 18 & $27.3 \%$ & 48 & $72.7 \%$ & & \\
\hline \multicolumn{8}{|l|}{ Differentiation } \\
\hline High & 15 & 8 & $53.3 \%$ & 7 & $46.7 \%$ & 248.5 & $<0.001^{\star \star \star}$ \\
\hline Medium & 146 & 63 & $43.2 \%$ & 83 & $56.8 \%$ & & \\
\hline Low & 424 & 134 & $31.6 \%$ & 290 & $68.4 \%$ & & \\
\hline \multicolumn{8}{|l|}{ Vessel invasion } \\
\hline Negative & 486 & 182 & $37.4 \%$ & 304 & $62.6 \%$ & 7.302 & $0.007^{\star \star *}$ \\
\hline Positive & 99 & 23 & $23.2 \%$ & 76 & $76.8 \%$ & & \\
\hline \multicolumn{8}{|l|}{ Nerve invasion } \\
\hline Negative & 504 & 188 & $37.3 \%$ & 316 & $62.7 \%$ & 8.159 & $0.004^{\star \star}$ \\
\hline Positive & 81 & 17 & $21.0 \%$ & 64 & $79.0 \%$ & & \\
\hline \multicolumn{8}{|l|}{ T stage } \\
\hline $\mathrm{T} 1$ & 76 & 34 & $44.7 \%$ & 42 & $55.3 \%$ & 586.7 & $<0.001^{\star \star \star}$ \\
\hline T2 & 85 & 39 & $45.9 \%$ & 46 & $54.1 \%$ & & \\
\hline T3 & 151 & 52 & $34.4 \%$ & 99 & $65.6 \%$ & & \\
\hline T4 & 273 & 80 & $29.3 \%$ & 193 & $70.7 \%$ & & \\
\hline \multicolumn{8}{|l|}{ N stage } \\
\hline NO & 228 & 106 & $46.5 \%$ & 122 & $53.5 \%$ & 596.6 & $<0.001^{\star \star \star}$ \\
\hline N1 & 105 & 35 & $33.3 \%$ & 70 & $66.7 \%$ & & \\
\hline N2 & 113 & 25 & $22.1 \%$ & 88 & $77.9 \%$ & & \\
\hline N3 & 139 & 39 & $28.1 \%$ & 100 & $71.9 \%$ & & \\
\hline \multicolumn{8}{|l|}{ TNM stage } \\
\hline । & 124 & 58 & $46.8 \%$ & 66 & $53.2 \%$ & 370.8 & $<0.001^{\star * *}$ \\
\hline$\|$ & 173 & 75 & $43.4 \%$ & 98 & $56.6 \%$ & & \\
\hline III & 288 & 72 & $25.0 \%$ & 216 & $75.0 \%$ & & \\
\hline
\end{tabular}

${ }^{a}$ Borrmann for 509 cases of advanced gastric cancer. ${ }^{*} P<0.05,{ }^{* *} P<0.01,{ }^{* * *} P<0001$.

DDX18 expression (Figure 5B). Therefore, the results indicated that DDX18 can promote the expression of microRNA-21.

A common pathway exists for the maturation of microRNAs, i.e., pre-microRNAs sheared and matured through the action of Drosha and DGCR8. However, the expression of microRNAs differed in different cells. Therefore, there must be molecules that can specifically recognize and bind pre-microRNAs and affect their expression levels. Combined with the above research, we found a relationship between DDX18 and microRNA-21. Next, through coimmunoprecipitation experiments, we found that DDX18 could interact with Drosha but could not interact with DGCR8 (Figure 5A). Therefore, we propose that DDX18 could affect the expression of microRNA-21 by interacting with Drosha (Figure 5D).

\section{Confirmation of the DDX18 $\rightarrow$ microRNA- 21 $\rightarrow$ PTEN/AKT Pathway}

In a previous study, we identified the role of DDX18 in gastric cancer cells and confirmed that DDX18 could promote the expression of microRNA-21. Therefore, the mechanism of action of microRNA-21 in gastric cancer cells was examined next. By analyzing the sequence of microRNA-21, we speculated that microRNA-21 might bind to the $3^{\prime}$-UTR of PTEN, promoting the degradation of PTEN mRNA and affecting related pathways downstream.

To further confirm the direct effect of miR-21-3p and PTEN, we used the dual luciferase gene reporter system to verify that PTEN was the target gene for miR-21-3p. The following plasmids were 
TABLE 2 | Univariate analysis of overall survival in 585 patients with gastric cancer.

\begin{tabular}{|c|c|c|c|}
\hline \multicolumn{4}{|c|}{ Univariate analysis } \\
\hline Clinicopathology & Hazard ratio & $95 \%$ confidence interval & $P$-value \\
\hline Age & 0.853 & $0.656-1.108$ & 0.234 \\
\hline Gender & 1.234 & $0.967-1.589$ & 0.089 \\
\hline Differentiation & 1.674 & $1.233-2.273$ & 0.001 \\
\hline Tumor location & 1.535 & $1.083-2.176$ & 0.016 \\
\hline Borrmann & 1.7 & $1.267-2.281$ & $<0.001$ \\
\hline Tumor size & 1.578 & $1.197-2.081$ & 0.001 \\
\hline Nerve invasion & 1.933 & $1.447-2.734$ & $<0.001$ \\
\hline Vessel invasion & 2.198 & $1.640-2.946$ & $<0.001$ \\
\hline T stage $(1,2 / 3,4)$ & 0.252 & $0.170-0.373$ & $<0.001$ \\
\hline$N$ stage $(0,1 / 2,3)$ & 0.242 & $0.185-0.315$ & $<0.001$ \\
\hline TNM stage $(1+2 / 3)$ & 0.237 & $0.178-0.314$ & $<0.001$ \\
\hline DDX18 expression & 2.605 & $2.208-3.345$ & $<0.001$ \\
\hline
\end{tabular}

TABLE 3 | Multivariate Cox regression analysis of overall survival in 585 patients with gastric cancer.

\begin{tabular}{|c|c|c|c|}
\hline \multicolumn{4}{|c|}{ Multivariate analysis } \\
\hline Clinicopathology & Hazard ratio & $95 \%$ confidence interval & $P$-value \\
\hline Differentiation & 1.12 & $0.815-1.538$ & 0.484 \\
\hline Tumor location & 1.132 & $0.856-1.498$ & 0.384 \\
\hline Borrmann & 1.02 & $0.746-1.349$ & 0.903 \\
\hline Tumor size & 1.176 & $0.887-1.559$ & 0.26 \\
\hline Nerve invasion & 1.203 & $0.850-1.701$ & 0.297 \\
\hline Vessel invasion & 1.145 & $0.828-1.582$ & 0.413 \\
\hline T stage $(1,2 / 3,4)$ & 0.607 & $0.366-1.006$ & 0.049 \\
\hline $\mathrm{N}$ stage $(0,1 / 2,3)$ & 0.453 & $0.296-0.694$ & $<0.001$ \\
\hline TNM stage $(1+2 / 3)$ & 0.411 & $0.306-0.533$ & $<0.001$ \\
\hline DDX18 expression & 1.513 & $1.150-1.991$ & 0.003 \\
\hline
\end{tabular}

cotransfected into $293 \mathrm{~T}$ cells: mimic miR-21-3p + PTEN 3'-UTR target clone plasmid, mimic NC + PTEN 3'-UTR target cloning plasmid, mimic miR-21-3p + negative control fluorescein enzyme plasmid, and mimic NC + negative control luciferase plasmid. The results showed that the miR-21-3p mimic could significantly inhibit the activity of luciferase $(P<0.01)$ and confirmed the direct effect of miR-21-3p and the PTEN 3'-UTR (Figure 5E).

We performed western blotting to confirm that DDX18 affects the PTEN/AKT signaling pathway by controlling the maturation of microRNA-21. We added microRNA-21 mimics, microRNA-21 inhibitor, and mTOR inhibitor to the AGS-shDDX18 cell line and finally added microRNA-21 mimics and mTOR inhibitor to detect the expression levels of DDX18, PTEN, p-AKT, and total AKT. The results showed that the expression of PTEN was inhibited again after readministration of the microRNA-21 mimics in the AGS cell line, and the phosphorylation of AKT was enhanced (Figure 6A).

Subsequently, we added microRNA-21 mimics, microRNA21 inhibitor, and mTOR inhibitor to the SGC-7901-OE cell line that had been transfected with DDX18 and finally added microRNA-21 inhibitor and mTOR inhibitor to detect the expression levels of DDX18, PTEN, p-AKT, and total AKT (Figure 6B). The results showed that the inhibition of PTEN was blocked by the addition of the microRNA-21 inhibitor, and the phosphorylation level of downstream AKT was significantly decreased. Therefore, DDX18 can indeed affect the expression of
PTEN by regulating microRNA-21, which in turn affects the phosphorylation of AKT and regulates the AKT signaling pathway. This result confirms our hypothesis (Figure 6C).

\section{Effect of DDX18 on Tumor Formation in Vitro}

AGS-sh-DDX18 cells and control AGS-sh-NC cells were used to induce subcutaneous tumor formation in the two groups of nude mice for 3 weeks. None of the mice in the two groups died during the experimental period. After 3 weeks of observation, the tumor-bearing nude mice were sacrificed, and the subcutaneous tumors were dissected. When the transplanted tumor was peeled from the skin, the tumor surface had a complete envelope with a clear boundary and slight adhesion to the surrounding tissue. We found that the average weight of the subcutaneous tumors in the control group was $1.57 \pm 0.56 \mathrm{~g}$, which was significantly different from that in the intervention group $(P=0.0003)$ and the interference group $(0.56 \pm 0.31 \mathrm{~g})$. DDX18 interference strongly inhibited the AGS cell subcutaneous tumorigenic ability in vivo, which was consistent with the results of the in vitro experiments (Figures 6D-G).

Then we detected the influence of DDX18 on the patient-derived xenograft (PDX) models. PDX models with low DDX18 expression were implanted in the mice. From the third day, group 1 was injected with PBS every day, while the corresponding group 2 was injected with the PTEN inhibitor SF1670. After 21 days, we found 
A

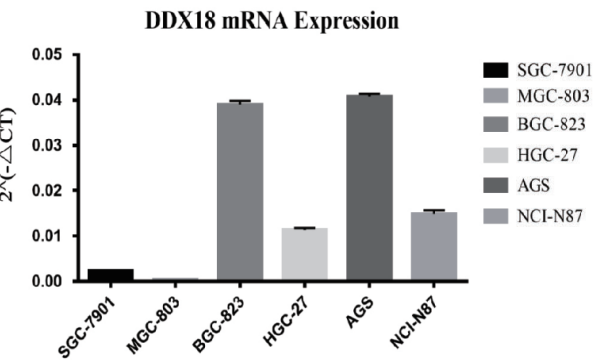

B
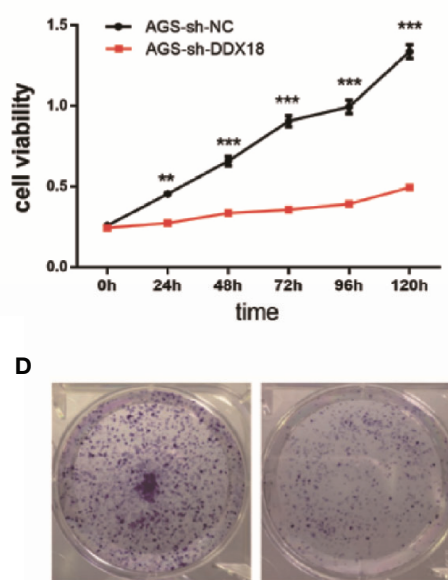

AGS-sh-NC

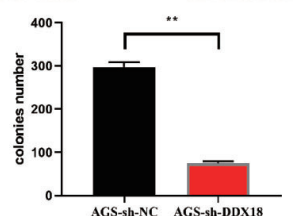

AGS-sh-DDX18
C

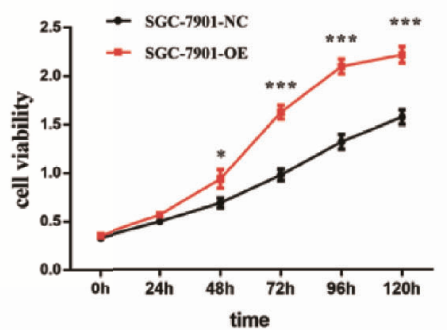

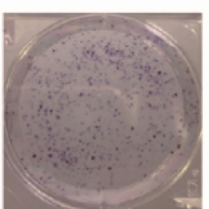

GS-sh-NC

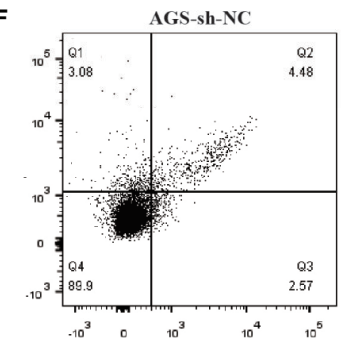

SGC-7901-NC

G

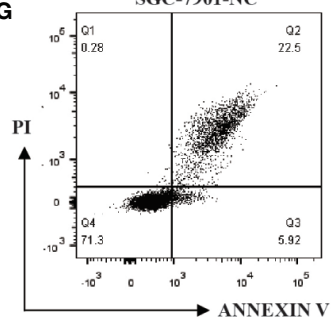

E
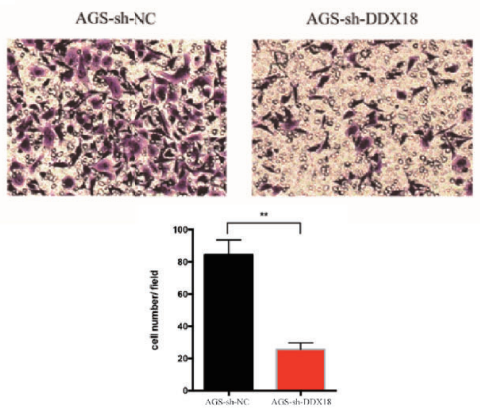

FIGURE 4 | Impact of DDX18 on gastric cancer cell lines. (A) DDX18 expression in gastric cancer cell lines. (B) DDX18 knockdown decreased the proliferation of gastric cancer cells. (C) DDX18 overexpression promotes the proliferation of gastric cancer cells. (D) DDX18 knockdown decreases clonal formation of gastric cancer cells. (E) DDX18 knockdown decreases invasion of gastric cancer cells. (F) DDX18 knockdown increases the apoptotic rate of gastric cancer cells. PI, propidium iodide. (G) DDX18 overexpression decreases the apoptotic rate of gastric cancer cells. PI, propidium iodide. 


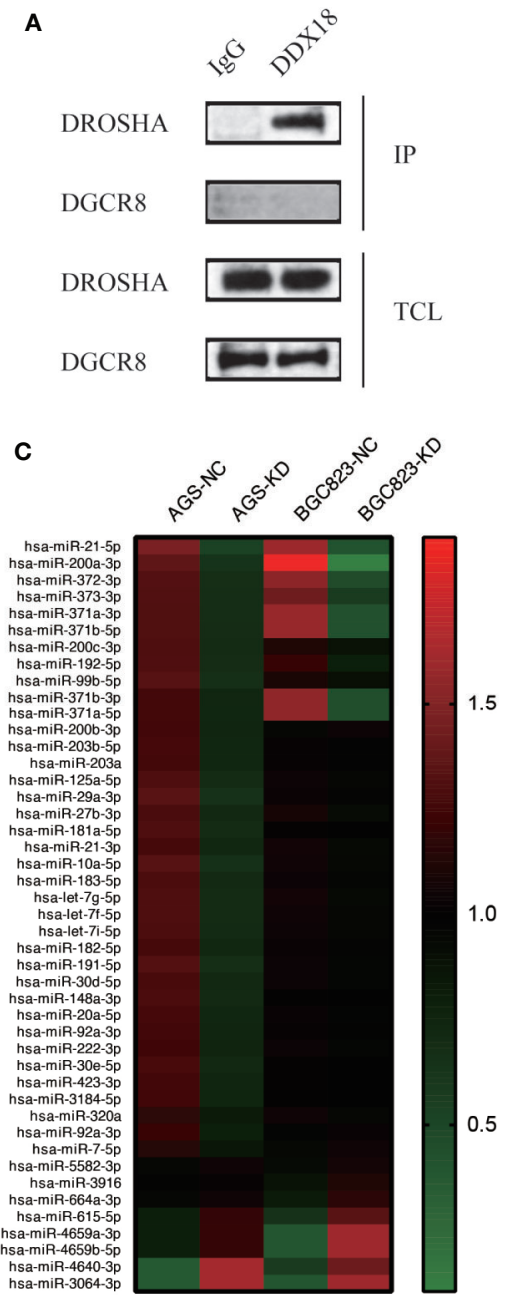

B

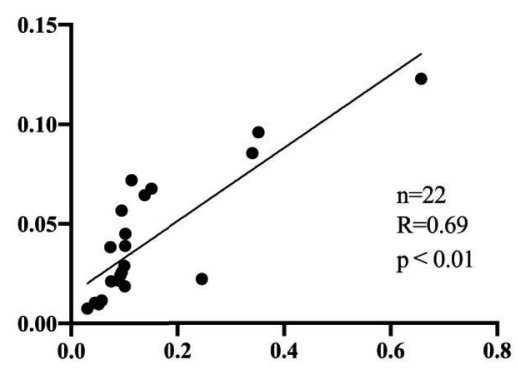

D

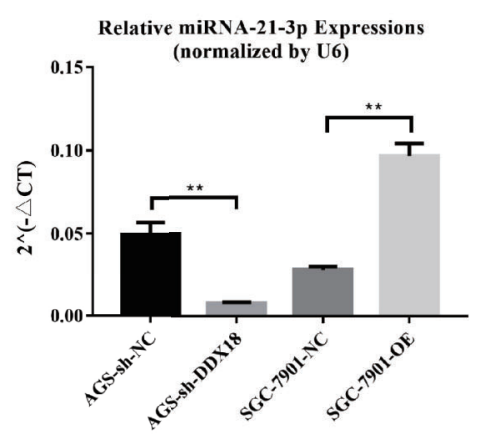

E

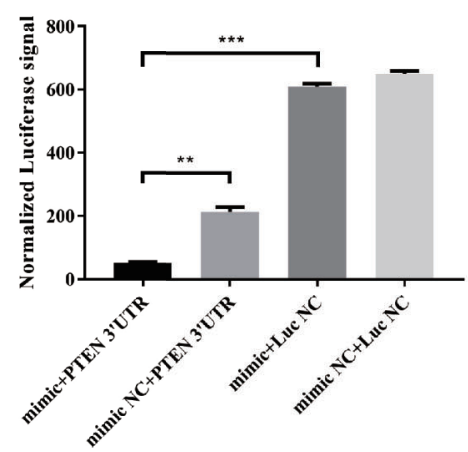

FIGURE 5 | Interaction of DDX18 and Drosha. (A) DDX18 interacts with Drosha but not DGCR8. (B) The expression of microRNA-21 is correlated with that of DDX18. (C) MicroRNA expressions in the DDX18-KD cells. (D) DDX18 could affect the expression of microRNA-21. (E) MicroRNA-21 could bind to the 3'untranslated region of PTEN and lead to degradation.

that the injection of SH1670 could effectively promote tumor growth (Figure 7A). IHC detection showed that, with the injection of SF1670, PTEN expression was significantly decreased, while the expression of P-AKT was reactivated (Figures 7B, C).

\section{DISCUSSION}

Gastric cancer is one of the most common malignant tumors worldwide. Data from the World Health Organization have shown that the number of new cases of gastric cancer ranked fifth among all malignant tumors, following lung cancer, breast cancer, colorectal cancer, and prostate cancer (1). In addition to the high incidence of gastric cancer, the mortality rate of gastric cancer ranks third among all malignant tumors worldwide because of its high degree of malignancy, hidden early symptoms, and relatively low sensitivity to radiotherapy and chemotherapy. Gastric cancer poses a serious threat to the lives and health of people worldwide. This disease is a major burden to patients and families and also consumes many public health resources. Therefore, further study of the relationship between the clinicopathological characteristics of gastric cancer and the prognosis of patients, exploration of effective treatment methods, and comprehensive improvements in the overall level of diagnosis and treatment of gastric cancer are urgently needed.

In our study, we first discussed the differential expression of DDX18 in gastric cancer tissues and adjacent tissues, identifying the specific high expression of DDX18 in the cancer samples. Combined with the analysis of clinical prognosis data, we found that DDX18 was positively correlated with the degree of 
A

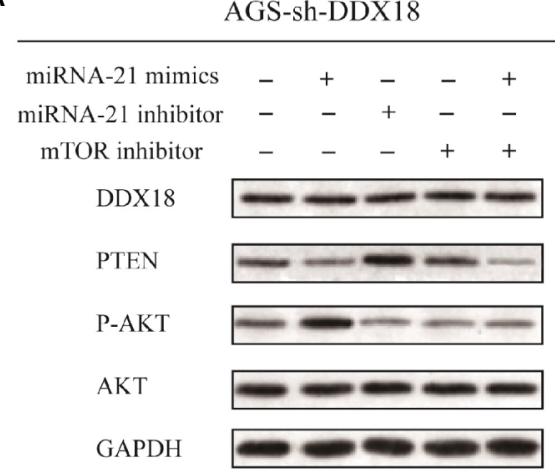

C

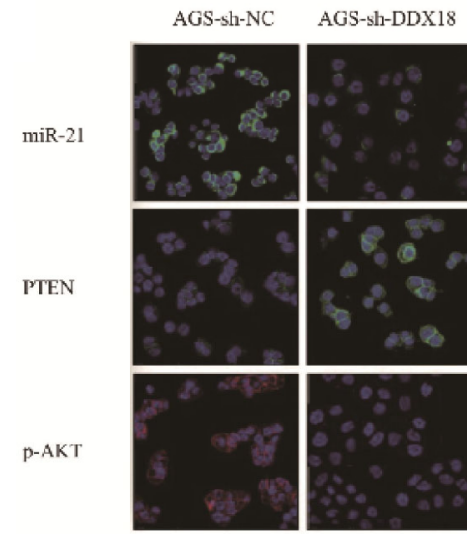

D

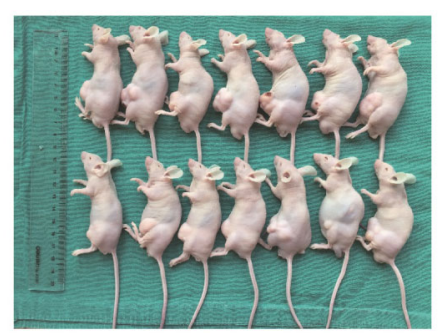

$\mathbf{F}$

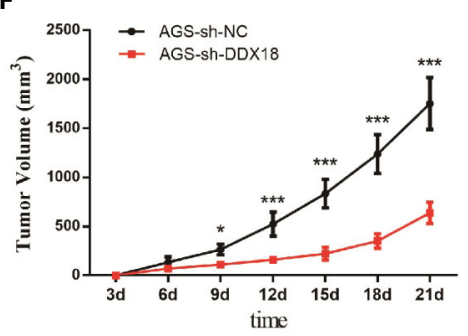

B

SGC-7901-OE

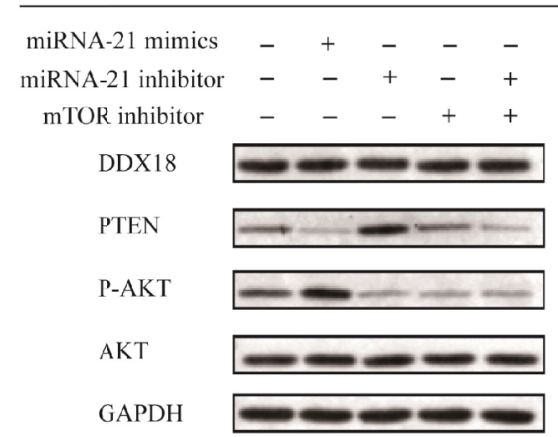

SGC-7901-NC SGC-7901-OE
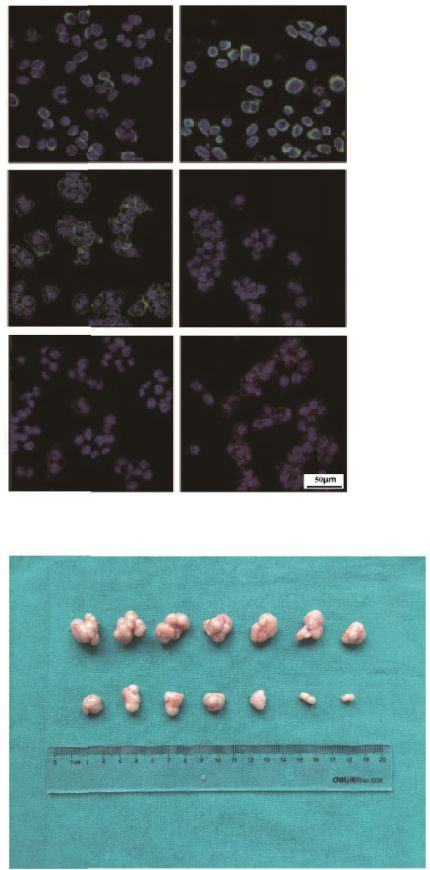

G

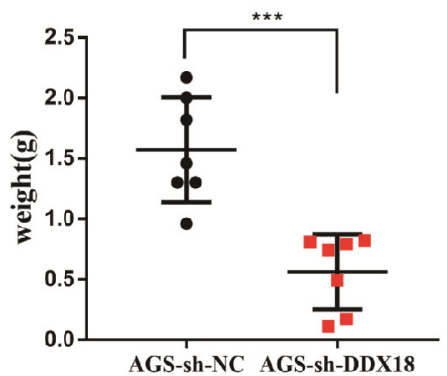

FIGURE 6 | The DDX18 $\rightarrow$ microRNA-21 $\rightarrow$ PTEN/AKT pathway. (A, B) DDX18 affects the PTEN/AKT signaling pathway by controlling the maturation of microRNA21. (C) DDX18 can also affect the expression of PTEN by regulating microRNA-21, which in turn affects the phosphorylation of AKT and regulates the AKT signaling pathway. (D-G) DDX18 interference strongly inhibited the AGS cell subcutaneous tumorigenic ability in vivo. ${ }^{\star} P<0.05,{ }^{\star * \star} P<0001$.

malignancy of gastric cancer and could significantly affect the prognosis of patients with gastric cancer. Further analysis showed that DDX18 could be applied as an independent risk factor for the prognosis of gastric cancer.
The role of ncRNAs, represented by microRNAs, in tumors, especially in gastric cancer, has been extensively studied in recent years (9-13). MicroRNAs can promote the degradation of RNA by targeting the $3^{\prime}$-UTR of the corresponding RNA, which can be 
A

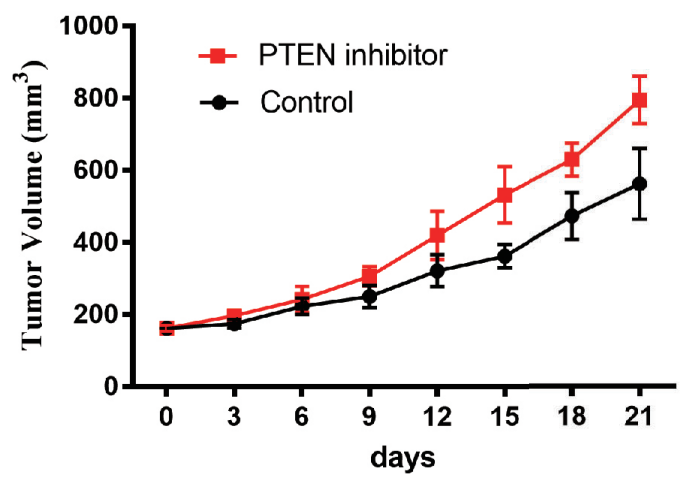

B

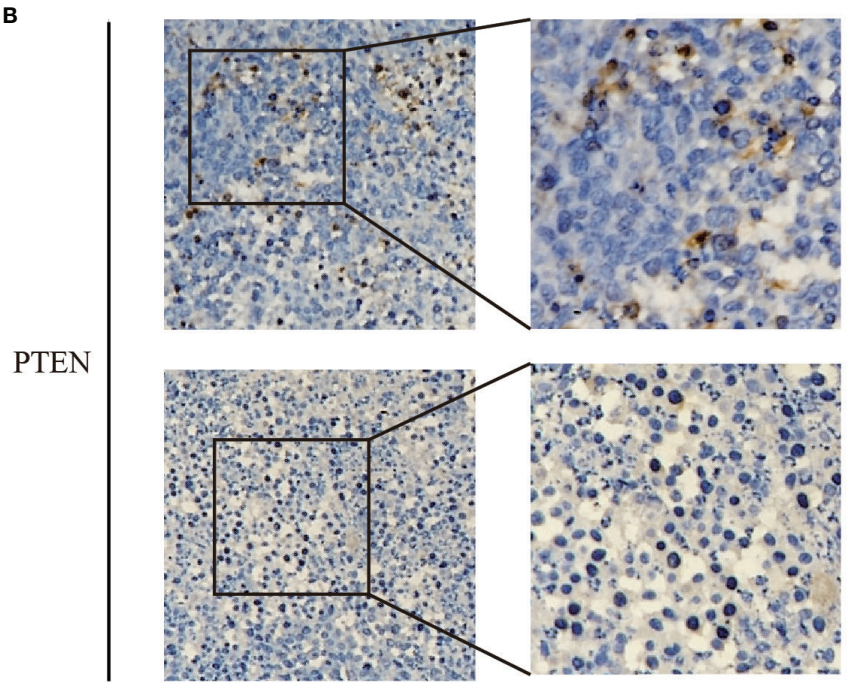

C

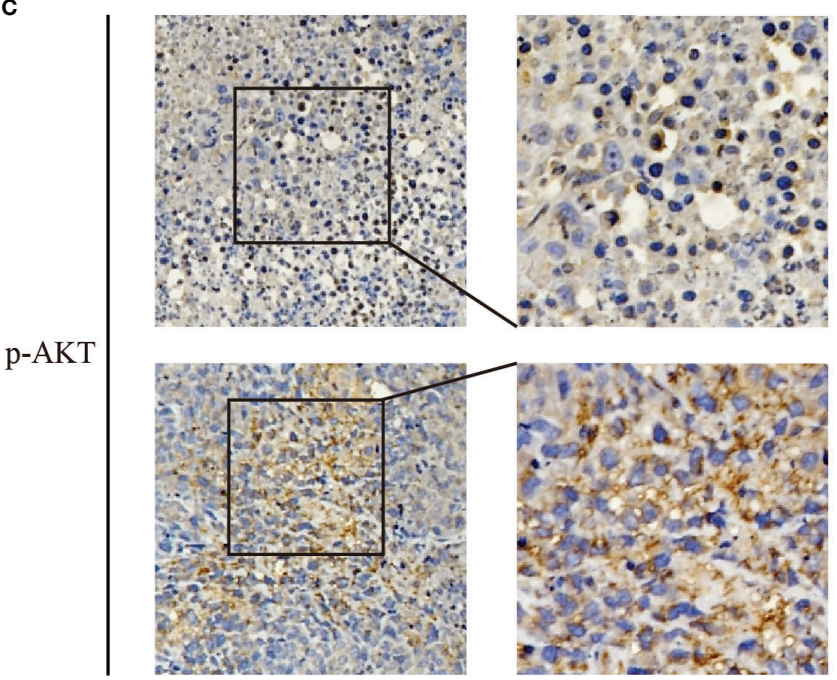

FIGURE 7 | The effect of DDX18 on gastric cancer patient-derived xenograft (PDX) models. (A) The growth curve of DDX18 on PDX models by injection of SF1670. (B, C) Immunohistochemistry of the effect on PDX models by injection of SF1670. 
regulated at the post-transcriptional level (14). At present, the maturation process of microRNAs is relatively clear. PremicroRNAs can be further matured through the shearing effect of Drosha and eventually become mature microRNAs (15). However, the regulation of the formation, maturation, and expression of microRNAs is still unknown. The RNA helicase family has RNA helicase activity. The protein family represented by the DDX family is an important part of this group.

Certain members of the DEAD-box helicase (DDX) family have been defined as cofactors to the Drosha complex, which controls the post-transcriptional maturation of a specific group of microRNAs. Drosha is an RNase III enzyme, which binds and cleaves double-stranded RNA without any sequence specificity (15). It is known that one nucleotide substitute of pri-microRNA in the stem-loop structure would inhibit Drosha-mediated modification, suggesting that the stem-loop structure should be critical for the enzymatic activity of Drosha (16). The Droshamediated processing depends on the unique complexity in the sequence or structure of microRNA, even though all primicroRNAs share some common features on one or more stem-loop structure. Some RNA-binding proteins, such as DDX18, serve as good candidates to identify this specificity through their selective interaction with targeted primicroRNAs. Furthermore, Drosha harbors no helicase activity compared with another RNase III endonuclease, Dicer, which is also involved in pre-microRNA processing (17). Taken together, potential interaction between DDX18 and Drosha not only confers microRNA-binding specificity but also assists in the generation of a desirable stem-loop structure for the indicated pri-microRNAs, followed by efficient cleavage.

In this study, we first found that DDX18 was associated with the malignancy of gastric cancer. Based on the clinical prognosis of patients, we confirmed that DDX18 is abnormally highly expressed in patients with advanced gastric cancer and is closely related to a poor prognosis. Therefore, we speculated that DDX18 may play an important role in the development and progression of gastric cancer. Furthermore, we performed in vivo and in vitro experiments to detect the function of DDX18 in gastric cancer. Our findings showed that DDX18 promoted tumor growth. Knockdown of DDX18 also substantially decreased gastric cancer cell migration and invasion. Further studies showed that DDX18 could regulate the maturation of microRNA-21 and then affect the function of the downstream PTEN/AKT signaling pathway. Both microRNA-21 and the PTEN/AKT signaling pathway have already been proved to play an important role in gastric cancer (18-20).

In a previous study on microRNAs, we focused on the downstream target genes and the functions of microRNAs but did not examine the upstream regulation of microRNA expression and maturation. This study provides a new direction for DDX family and

\section{REFERENCES}

1. Bray F, Ferlay J, Soerjomataram I, Siegel RL, Torre LA, Jemal A. Global cancer statistics 2018: GLOBOCAN estimates of incidence and mortality worldwide for 36 cancers in 185 countries. CA Cancer J Clin (2018) 68:394-424. doi: $10.3322 /$ caac. 21492
microRNA research. Moreover, we provide a preliminary experimental basis and theoretical basis for the future study of DDX18 as a new target for the treatment of gastric cancer.

\section{DATA AVAILABILITY STATEMENT}

The original contributions presented in the study are included in the article/Supplementary Material. Further inquiries can be directed to the corresponding authors.

\section{ETHICS STATEMENT}

The studies involving human participants were reviewed and approved by Ethics Committee of Renji Hospital, School of Medicine, Shanghai JiaoTong University. The patients/ participants provided their written informed consent to participate in this study. The animal study was reviewed and approved by Ethics Committee of Renji Hospital, School of Medicine, Shanghai JiaoTong University.

\section{AUTHOR CONTRIBUTIONS}

CZ and S-HK designed the study. YZ, FY, QL, BN, S-WB, and $\mathrm{J}-\mathrm{HC}$ performed the in vitro and in vivo experiments. $\mathrm{YZ}$ and $\mathrm{FY}$ wrote this manuscript. $\mathrm{H}-\mathrm{KY}$ provided valuable suggestions for this research. YZ provided help of bioinformatics analysis. CZ provided the clinical samples and prognostic data. CZ and S-HK provided the funding that supported this research. All authors contributed to the article and approved the submitted version.

\section{FUNDING}

This research was supported by grants from the National Natural Science Foundation of China (nos. 81602062 and 81811540415).

\section{SUPPLEMENTARY MATERIAL}

The Supplementary Material for this article can be found online at: https://www.frontiersin.org/articles/10.3389/fonc.2020. 598238/full\#supplementary-material

SUPPLEMENTARY FIGURE 1 | Impact of DDX18 on BGC-823 cell lines. (A) DDX18 knockdown decrease clonal formation of BGC-823 cell line. (B) DDX18 knockdown decrease invasion of BGC-823 cell line.

2. Lee RC, Feinbaum RL, Ambros V, The C. elegans heterochronic gene lin-4 encodes small RNAs with antisense complementarity to lin-14. Cell (1993) 75:843-54. doi: 10.1016/0092-8674(93)90529-Y

3. Abdelhaleem M, Maltais L, Wain H, The human DDX. and DHX gene families of putative RNA helicases. Genomics (2003) 81:618-22. doi: 10.1016/ S0888-7543(03)00049-1 
4. Yin J, Park G, Lee JE, Choi EY, Park JY, Kim TH, et al. DEAD-box RNA helicase DDX23 modulates glioma malignancy via elevating miR-21 biogenesis. Brain (2015) 138:2553-70. doi: 10.1093/brain/awv167

5. Hashemi V, Masjedi A, Hazhir-Karzar B, Tanomand A, Shotorbani SS, Hojjat-Farsangi M, et al. The role of DEAD-box RNA helicase p68 (DDX5) in the development and treatment of breast cancer. J Cell Physiol (2019) 234:5478-87. doi: 10.1002/jcp.26912

6. Wang X, Li P, Wang C, Zhang D, Zeng L, Liu X, et al. DEAD-box RNA Helicase 39 Promotes Invasiveness and Chemoresistance of ER-positive Breast Cancer. J Cancer (2020) 11:1846-58. doi: 10.7150/jca.37247

7. Gregory RI, Chendrimada TP, Shiekhattar R. MicroRNA biogenesis: isolation and characterization of the microprocessor complex. Methods Mol Biol (2006) 342:33-47. doi: 10.1385/1-59745-123-1:33

8. Calin GA, Liu CG, Sevignani C, Ferracin M, Felli N, Dumitru CD, et al. MicroRNA profiling reveals distinct signatures in B cell chronic lymphocytic leukemias. Proc Natl Acad Sci USA (2004) 101:11755-60. doi: 10.1073/ pnas.0404432101

9. Liu Y, Xu Y, Xiao F, Zhang J, Wang Y, Yao Y, et al. Comprehensive Analysis of a circRNA-miRNA-mRNA Network to Reveal Potential InflammationRelated Targets for Gastric Adenocarcinoma. Mediators Inflammation (2020) 2020:9435608. doi: 10.1155/2020/9435608

10. Schaalan M, Mohammad W, Fathy S. MiRNA-200c, MiRNA-139 and ln RNA $\mathrm{H} 19$; new predictors of treatment response in H-pylori- induced gastric ulcer or progression to gastric cancer. Microb Pathog (2020) 149:104442. doi: 10.1016/j.micpath.2020.104442

11. Xie S, Chang Y, Jin H, Feng Y, Xu Y, Yan X, et al. Non-coding RNAs in gastric cancer. Cancer Lett (2020) 493:55-70. doi: 10.1016/j.canlet.2020.06.022

12. Zhao H, Zheng Y, You J, Xiong J, Ying S, Xie L, et al. Tumor suppressor role of miR-876-5p in gastric cancer. Oncol Lett (2020) 20:1281-7. doi: 10.3892/ ol.2020.11680

13. Wan G, Liu Y, Han C, Zhang X, Lu X. Noncoding RNAs in DNA repair and genome integrity. Antioxid Redox Signal (2014) 20:655-77. doi: 10.1089/ ars.2013.5514
14. Zhang X, Lu X. Posttranscriptional regulation of miRNAs in the DNA damage response. RNA Biol (2011) 8:960-3. doi: 10.4161/rna.8.6.17337

15. Lund E, Dahlberg JE. Substrate selectivity of exportin 5 and Dicer in the biogenesis of microRNAs. Cold Spring Harb Symp Quant Biol (2006) 71:5966. doi: 10.1101/sqb.2006.71.050

16. Slezak-Prochazka I, Durmus S, Kroesen BJ, van den Berg A. MicroRNAs, macrocontrol: regulation of miRNA processing. RNA (2010) 16:1087-95. doi 10.1261/rna.1804410

17. Welker NC, Maity TS, Ye X, Aruscavage PJ, Krauchuk AA, Liu Q, et al. Dicer's helicase domain discriminates dsRNA termini to promote an altered reaction mode. Mol Cell (2011) 41:589-99. doi: 10.1016/j.molcel.2011.02.005

18. Uozaki H, Morita S, Kumagai A, Aso T, Soejima Y, Takahashi Y, et al. Stromal miR-21 is more important than miR-21 of tumour cells for the progression of gastric cancer. Histopathology (2014) 65:775-83. doi: 10.1111/his.12491

19. Wang P, Guan Q, Zhou D, Yu Z, Song Y, Qiu W. miR-21 Inhibitors Modulate Biological Functions of Gastric Cancer Cells via PTEN/PI3K/mTOR Pathway. DNA Cell Biol (2018) 37:38-45. doi: 10.1089/dna.2017.3922

20. Qiang Z, Meng L, Yi C, Yu L, Chen W, Sha W. Curcumin regulates the miR21/PTEN/Akt pathway and acts in synergy with PD98059 to induce apoptosis of human gastric cancer MGC-803 cells. J Int Med Res (2019) 47:1288-97. doi: $10.1177 / 0300060518822213$

Conflict of Interest: The authors declare that the research was conducted in the absence of any commercial or financial relationships that could be construed as a potential conflict of interest.

Copyright (c) 2021 Zhang, Yu, Ni, Li, Bae, Choi, Yang, Kong and Zhu. This is an openaccess article distributed under the terms of the Creative Commons Attribution License (CC BY). The use, distribution or reproduction in other forums is permitted, provided the original author(s) and the copyright owner(s) are credited and that the original publication in this journal is cited, in accordance with accepted academic practice. No use, distribution or reproduction is permitted which does not comply with these terms. 